\title{
Jacobi Last Multiplier and Lie Symmetries: A Novel Application of an Old Relationship
}

\author{
$M C N U C C I$ \\ Dipartimento di Matematica e Informatica, Università di Perugia, 06123 Perugia, Italy \\ E-mail: nucci@unipg.it
}

Received March, 2005; Accepted March, 2005

\begin{abstract}
After giving a brief account of the Jacobi last multiplier for ordinary differential equations and its known relationship with Lie symmetries, we present a novel application which exploits the Jacobi last multiplier to the purpose of finding Lie symmetries of first-order systems. Several illustrative examples are given.
\end{abstract}

\section{Introduction}

The late Summer of 1843 sees Carl Gustav Jacob Jacobi travelling south: his final destination is Italy. The poor condition of his health has forced him to make this journey leaving behind his beloved family. Although his health seems to improve thanks maybe to the better climate, Jacobi is not only missing his family but also finds himself longing to discuss mathematics with Italian mathematicians [32]. One occasion that presents itself is the Congress of the Italian Scientists [1] in Lucca. Jacobi wrote to his wife that he had the audacity to give an impromptu lecture in French because he thought that it was a question of courtesy to make a communication of some sort, but he had neither the time nor the peace of mind necessary to write anything [32]. The summary of that lecture was published a year later in an Italian journal". Its title in English is "On the principle of the last multiplier and its use as a new broad principle of mechanics" [25]. This is the first appearance in the literature of a new mathematical object which is nowadays known as the Jacobi last multiplier ${ }^{2}$. The really extensive work on the last multiplier was then published in Latin in Crelle's journal as a very long article divided into three parts ([26], $[27])$ and filled with various examples, e.g. his problem of three bodies which attract each other with forces proportional to the cube of the inverse of their distance and move on a line [46]. Note that in his lectures on dynamics [28] - published posthumously - which he had delivered in the Winter of 1842-1843 at the University of Berlin several chapters

Copyright (C) 2005 by $M$ C Nucci

\footnotetext{
${ }^{1}$ That journal was published in Rome and terminated in 1868.

${ }^{2}$ Actually, during his journey with Bessel to England in 1842 [32], Jacobi went to Manchester and at a meeting of the British Association for the Advancement of Science presented a communication in English entitled "On a new general principle of analytical mechanics" which in the same year was published in French by the Paris Academy [24]. In that paper he announced his new principle of Mechanics and mentioned a last multiplier without any mathematical detail contrarily to what is reported in [25].
} 
are devoted to the last multiplier and its applications in Classical Mechanics. After Jacobi's untimely death in 1851 his last multiplier had been used to find first integrals, e.g. for a certain second-order ordinary differential equation [34], but it was when Sophus Lie found a link with the symmetries that bear his name [36] that many authors studied the Jacobi last multiplier in different contexts: from searching for its generalization [13]; to exploiting all the possible relationships it has with first integrals and Lie symmetries ([9], [56]); from determining a Lagrangian formulation for systems of second-order ordinary differential equations ([58], [38], [29]); to searching for steady compressible flows of perfect gases [51] or applying it to the statistical mechanics of dissipative systems [14]; from identifying the time-dependent probability density as its analogue in Quantum Mechanics [33]; to the ubiquitous relationship between inverse Jacobi last multiplier and limit cycles [2]; from finding first integrals of various systems ([18], [54], [50]); to deducing nonlocal Lie symmetries [48].

In the present paper we show a novel application of the Jacobi last multiplier in the quest for Lie symmetries admitted by ordinary differential equations.

Lie group analysis is indeed the most powerful tool to find the general solution of ordinary differential equations. Any known technique of integration ${ }^{3}$ can be shown to be a particular case of a general method of integration based on the derivation of the continuous group of symmetries admitted by the differential equation, i.e. the Lie symmetry algebra, which can be easily derived by a straightforward although lengthy procedure. As computer algebra software becomes widely used, the integration of systems of ordinary differential equations by means of Lie group analysis is becoming easier to perform. A major drawback of Lie's method is that it is useless when applied to systems of $M$ first-order equations ${ }^{4}$, because they admit an infinite number of symmetries, and there is no systematic way to find even an one-dimensional Lie symmetry algebra, apart from trivial groups like translations in time admitted by autonomous systems. One may try to derive an admitted $M$-dimensional solvable Lie symmetry algebra by making an ansatz on the form of its generators, but when successful (rarely!) it is just a lucky guess.

However, in [43] we have remarked that any system of $n$ first-order equations could be transformed into an equivalent system where at least one of the equations is of secondorder. Then the admitted Lie symmetry algebra is no longer infinite-dimensional and nontrivial symmetries of the original system can be retrieved [43]. This idea has been successfully applied in several instances ([43], [57], [47], [44], [49], [35], [45], [15], [53]). Also in [39] we have shown that first integrals can be obtained by Lie group analysis even if the system under study does not come from a variational problem, i.e. without making use of Noether's theorem [40]. The key is to solve the linear parabolic determining equation which comes after the reduction method has been applied; the characteristic curves correspond to first integrals. We have used this method for finding first integrals in [39], [44] and [35].

\footnotetext{
${ }^{3}$ We mean those taught in most undergraduate courses on ordinary differential equations.

${ }^{4}$ Any undergraduate science/engineering student knows that an $n$ th-order ordinary differential equation can be transformed into an equivalent system of $n$ first-order equations. Less well-known to students but common knowledge among experts in Lie group analysis is the dramatic consequence that that transformation has on the dimension of the admitted Lie symmetry algebra. In fact, while the maximal Lie symmetry algebra admitted by a single $n$ th-order equation is finite [16], the dimension of the Lie symmetry algebra admitted by a system of $n$ first-order equations is infinite.
} 
The outline of the present paper is as follows. In Section 2 we recall the classical "old" relationship between the Jacobi last multiplier and Lie symmetries and provide an expository example of a linearizable equation which yields a "plenitude" of first integrals. In Section 3 we present a new application of the Jacobi last multiplier in order to raise the order of the system under study and thereby allowing for more Lie symmetries to be found: several examples are given.

\section{Jacobi last multiplier and Lie symmetries}

The method of the Jacobi last multiplier ([25], [26], [27], [28]) provides a means to determine all the solutions of the partial differential equation

$$
\mathcal{A} f=\sum_{i=1}^{n} a_{i}\left(x_{1}, \ldots, x_{n}\right) \frac{\partial f}{\partial x_{i}}=0
$$

or its equivalent associated Lagrange's system

$$
\frac{\mathrm{d} x_{1}}{a_{1}}=\frac{\mathrm{d} x_{2}}{a_{2}}=\ldots=\frac{\mathrm{d} x_{n}}{a_{n}} .
$$

In fact, if one knows the Jacobi last multiplier and all but one of the solutions, then the last solution can be obtained by a quadrature. The Jacobi last multiplier, $M$, is given by

$$
\frac{\partial\left(f, \omega_{1}, \omega_{2}, \ldots, \omega_{n-1}\right)}{\partial\left(x_{1}, x_{2}, \ldots, x_{n}\right)}=M \mathcal{A} f
$$

where

$$
\frac{\partial\left(f, \omega_{1}, \omega_{2}, \ldots, \omega_{n-1}\right)}{\partial\left(x_{1}, x_{2}, \ldots, x_{n}\right)}=\operatorname{det}\left[\begin{array}{ccc}
\frac{\partial f}{\partial x_{1}} & \cdots & \frac{\partial f}{\partial x_{n}} \\
\frac{\partial \omega_{1}}{\partial x_{1}} & & \frac{\partial \omega_{1}}{\partial x_{n}} \\
\vdots & & \vdots \\
\frac{\partial \omega_{n-1}}{\partial x_{1}} & \cdots & \frac{\partial \omega_{n-1}}{\partial x_{n}}
\end{array}\right]=0
$$

and $\omega_{1}, \ldots, \omega_{n-1}$ are $n-1$ solutions of (2.1) or, equivalently, first integrals of (2.2) independent of each other. This means that $M$ is a function of the variables $\left(x_{1}, \ldots, x_{n}\right)$ and depends on the chosen $n-1$ solutions, in the sense that it varies as they vary. The essential properties of the Jacobi last multiplier are:

a) If one selects a different set of $n-1$ independent solutions, $\eta_{1}, \ldots, \eta_{n-1}$, of equation

(2.1), then the corresponding last multiplier $N$ is linked to $M$ by the relationship:

$$
N=M \frac{\partial\left(\eta_{1}, \ldots, \eta_{n-1}\right)}{\partial\left(\omega_{1}, \ldots, \omega_{n-1}\right)} .
$$

b) Given a nonsingular transformation of variables,

$$
\tau: \quad\left(x_{1}, x_{2}, \ldots, x_{n}\right) \longrightarrow\left(x_{1}^{\prime}, x_{2}^{\prime}, \ldots, x_{n}^{\prime}\right),
$$


then the last multiplier $M^{\prime}$ of $\mathcal{A}^{\prime} F=0$ is given by:

$$
M^{\prime}=M \frac{\partial\left(x_{1}^{\prime}, x_{2}^{\prime}, \ldots, x_{n}^{\prime}\right)}{\partial\left(x_{1}, x_{2}, \ldots, x_{n}\right)},
$$

where $M$ obviously comes from the $n-1$ solutions of $\mathcal{A} F=0$ which correspond to those chosen for $\mathcal{A}^{\prime} F=0$ through the inverse transformation $\tau^{-1}$.

c) One can prove that each multiplier $M$ is a solution of the following linear partial differential equation:

$$
\sum_{i=1}^{n} \frac{\partial\left(M a_{i}\right)}{\partial x_{i}}=0 ;
$$

vice versa every solution $M$ of this equation is a Jacobi last multiplier.

d) If one knows two Jacobi last multipliers, $M_{1}$ and $M_{2}$, of equation (2.1), then their ratio is a solution $\omega$ of (2.1) or, equivalently, a first integral of (2.2). Naturally the ratio may be quite trivial, namely a constant. Vice versa the product of a multiplier $M_{1}$ times any solution $\omega$ yields another last multiplier $M_{2}=M_{1} \omega$.

In its original formulation the method of the Jacobi last multiplier required almost complete knowledge of the system, (2.1) or (2.2), under consideration. Since the existence of a solution/first integral is consequent upon the existence of symmetry, an alternate formulation in terms of symmetries was provided by Lie [37]. A clear treatment of the formulation in terms of solutions/first integrals and symmetries is given by Bianchi [3]. If we know $n-1$ symmetries of $(2.1) /(2.2)$, say

$$
\Gamma_{i}=\sum_{j=1}^{n} \xi_{i j}\left(x_{1}, \ldots, x_{n}\right) \partial_{x_{j}}, \quad i=1, n-1,
$$

Jacobi's last multiplier is given by $M=\Delta^{-1}$, provided that $\Delta \neq 0$, where

$$
\Delta=\operatorname{det}\left[\begin{array}{ccc}
a_{1} & \cdots & a_{n} \\
\xi_{1,1} & & \xi_{1, n} \\
\vdots & & \vdots \\
\xi_{n-1,1} & \cdots & \xi_{n-1, n}
\end{array}\right]
$$

There is an obvious corollary to the results of Jacobi mentioned above. In the case that there exists a constant multiplier, the determinant is a first integral. This result is potentially very useful in the search for first integrals of systems of ordinary differential equations. The differential equation to be solved for the Jacobi last multiplier is

$$
\frac{\mathrm{d} \log (M)}{\mathrm{d} t}+\sum_{i=1}^{n} \frac{\partial W_{i}}{\partial w_{i}}=0,
$$

where $M$ is the multiplier and the equation of motion has components

$$
\dot{w}_{i}=W_{i}\left(w_{1}, \ldots, w_{n}\right)
$$


in which overdot denotes differentiation with respect to an independent variable, say $t$. Consequently, if each component of the vector field of the equation of motion is missing the variable associated with that component, i.e., $\partial W_{i} / \partial w_{i}=0$, the last multiplier is a constant. This feature was put to good use with the Euler-Poinsot system [48] and the Kepler problem [50]. Note that equation (2.7) implies that the Jacobi last multiplier, $M$, is equal to

$$
M=\exp \left(-\int \sum_{i=1}^{n} \frac{\partial W_{i}}{\partial w_{i}} d t\right) .
$$

\subsection{A linearizable equation given by Painlevé}

We consider a second-order ordinary differential equation which was given by Painlevé [52] and cited by Ince [23] as an example of an equation which does not possess the Painlevé property $^{5}$ :

$$
w^{\prime \prime}=w^{\prime 2}\left(w \frac{2 k^{2} w^{2}-1-k^{2}}{\left(1-w^{2}\right)\left(1-k^{2} w^{2}\right)}-\frac{1}{\lambda \sqrt{\left(1-w^{2}\right)\left(1-k^{2} w^{2}\right)}}\right) .
$$

Recently a modified version of equation (2.10) was considered by Calogero [6] to show that it features many periodic solutions and that all of its nonsingular solutions are periodic. After a cosmetic rescaling, $u=w / \lambda$, here we show that in the case when $k=1$ (also considered in $[6])$ equation $(2.10)$, i.e.,

$$
u^{\prime \prime}=u^{\prime 2} \frac{2 u+1}{u^{2}-\lambda^{2}},
$$

admits an eight-dimensional Lie symmetry algebra ${ }^{6}$ generated by the following eight operators:

$$
\begin{aligned}
& X_{1}=-\left(\frac{u+\lambda}{u-\lambda}\right)^{\frac{1}{2 \lambda}}\left[x \partial_{x}+\left(\lambda^{2}-u^{2}\right) \partial_{u}\right] \\
& X_{2}=-\left(\frac{u+\lambda}{u-\lambda}\right)^{\frac{1}{2 \lambda}} \partial_{x} \\
& X_{3}=\left(\frac{u-\lambda}{u+\lambda}\right)^{\frac{1}{2 \lambda}}\left(u^{2}-\lambda^{2}\right) x \partial_{u} \\
& X_{4}=\left(\frac{u-\lambda}{u+\lambda}\right)^{\frac{1}{2 \lambda}}\left(u^{2}-\lambda^{2}\right) \partial_{u} \\
& X_{5}=-x^{2} \partial_{x}+x\left(u^{2}-\lambda^{2}\right) \partial_{u} \\
& X_{6}=\left(u^{2}-\lambda^{2}\right) \partial_{u} \\
& X_{7}=\partial_{x} \\
& X_{8}=x \partial_{x}
\end{aligned}
$$

${ }^{5}$ Here prime denotes differentiation with respect to $x$.

${ }^{6}$ This symmetry algebra is isomorphic to $\operatorname{sl}(3, \mathbb{R})([37],[16])$. 
which means that equation (2.11) is linearizable [37] by means of a point transformation ${ }^{7}$. In order to find the linearizing transformation we have to look for a two-dimensional abelian intransitive subalgebra and, following Lie's classification of two-dimensional algebras in the real plane [37], we have to transform the subalgebra into the canonical form

$$
\partial_{\tilde{u}}, \quad \tilde{x} \partial_{\tilde{u}}
$$

with $\tilde{u}$ and $\tilde{x}$ the new dependent and independent variables, respectively. We find that one such subalgebra is that generated by $X_{2}$ and $X_{7}$. Then it is easy to derive that

$$
\tilde{x}=\left(\frac{u-\lambda}{u+\lambda}\right)^{\frac{1}{2 \lambda}}, \quad \tilde{u}=x\left(\frac{u-\lambda}{u+\lambda}\right)^{\frac{1}{2 \lambda}}
$$

and equation (2.11) becomes

$$
\frac{\mathrm{d}^{2} \tilde{u}}{\mathrm{~d} \tilde{x}^{2}}=0
$$

The general solution is trivially $\tilde{u}=a_{1} \tilde{x}+a_{2}$, with $a_{1}$ and $a_{2}$ arbitrary constants, and finally thanks to transformation (2.14) the general solution of equation (2.11) is:

$$
u=\lambda \frac{\left(x-a_{1}\right)^{2 \lambda}+a_{2}^{2 \lambda}}{\left(x-a_{1}\right)^{2 \lambda}-a_{2}^{2 \lambda}} .
$$

In order to apply the method of the Jacobi last multiplier equation (2.11) is written as the system of two autonomous first-order ordinary differential equations:

$$
\begin{aligned}
u^{\prime} & =u_{x}, \\
u_{x}^{\prime} & =u_{x}^{2} \frac{2 u+1}{u^{2}-\lambda^{2}}
\end{aligned}
$$

with $u_{x}$ a new obvious dependent variable. Of course we have trivially to construct the first prolongations of the generators (2.12) in order for each of them to generate a Lie symmetry of system (2.17). For example the first prolongation of $X_{1}$ is

$$
X_{1}=X_{1}+\left(\frac{u+\lambda}{u-\lambda}\right)^{\frac{1}{2 \lambda}} u_{x} \frac{x u_{x}+2 \lambda^{2} u-2 u^{3}}{\lambda^{2}-u^{2}} \partial_{u_{x}}
$$

The Jacobi last multiplier of system (2.17) has to satisfy equation (2.7), i.e.,

$$
\frac{\mathrm{d} \log (M)}{\mathrm{d} x}=2 u_{x} \frac{2 u+1}{\lambda^{2}-u^{2}} .
$$

Note that, although system (2.17) is autonomous, the dependent variable $x$ appears in nearly all the generators (2.12). This means that the corresponding matrices as given in

\footnotetext{
${ }^{7}$ We do not know if the linear nature of equation (2.11) has been already shown elsewhere. Here our goal is to show that the known relationship between the Jacobi last multiplier and Lie symmetries yields first integrals of equation (2.11).
} 
(2.6) must have three columns and consequently there are twenty-eight possible determinants to be calculated. By way of illustration the matrix that we obtain with the choice of $X_{1}$ and $X_{3}$ is

$$
C_{13}=\left[\begin{array}{ccc}
1 & u_{x} & u_{x}^{2} \frac{2 u+1}{u^{2}-\lambda^{2}} \\
-\left(\frac{u+\lambda}{u-\lambda}\right)^{\frac{1}{2 \lambda}} x & \left(\frac{u+\lambda}{u-\lambda}\right)^{\frac{1}{2 \lambda}}\left(u^{2}-\lambda^{2}\right) & \left(\frac{u+\lambda}{u-\lambda}\right)^{\frac{1}{2 \lambda}} u_{x} \frac{x u_{x}+2 \lambda^{2} u-2 u^{3}}{\lambda^{2}-u^{2}} \\
0 & \left(\frac{u-\lambda}{u+\lambda}\right)^{\frac{1}{2 \lambda}}\left(u^{2}-\lambda^{2}\right) x & \left(\frac{u-\lambda}{u+\lambda}\right)^{\frac{1}{2 \lambda}}\left(2 x u u_{x}+x u_{x}+u^{2}-\lambda^{2}\right)
\end{array}\right] .
$$

First integrals of system (2.17) are then obviously obtained by taking any ratio of two determinants which are not null. With the use of Maple 9 it is easy to find that the determinants which differ from zero are the following ${ }^{8}$ :

$$
\begin{aligned}
& \Delta_{13}=\left(u^{2}-\lambda^{2}+x u_{x}\right)^{2} \\
& \Delta_{14}=\left(u^{2}-\lambda^{2}+x u_{x}\right) u_{x} \\
& \Delta_{15}=\left(\frac{u+\lambda}{u-\lambda}\right)^{\frac{1}{2 \lambda}} \frac{\left(u^{2}-\lambda^{2}+x u_{x}\right)^{3}}{u^{2}-\lambda^{2}} \\
& \Delta_{17}=\left(\frac{u+\lambda}{u-\lambda}\right)^{\frac{1}{2 \lambda}} \frac{u_{x}^{2}\left(u^{2}-\lambda^{2}+x u_{x}\right)}{\lambda^{2}-u^{2}} \\
& \Delta_{18}=\left(\frac{u+\lambda}{u-\lambda}\right)^{\frac{1}{2 \lambda}} \frac{u_{x}\left(u^{2}-\lambda^{2}+x u_{x}\right)^{2}}{\lambda^{2}-u^{2}} \\
& \Delta_{23}=\Delta_{14} \\
& \Delta_{24}=u_{x}^{2} \\
& \Delta_{25}=\Delta_{18} \\
& \Delta_{27}=\left(\frac{u+\lambda}{u-\lambda}\right)^{\frac{1}{2 \lambda}} \frac{u_{x}^{3}}{\lambda^{2}-u^{2}} \\
& \Delta_{28}=\Delta_{17} \\
& \Delta_{34}=-\left(\frac{u-\lambda}{u+\lambda}\right)^{\frac{1}{\lambda}}\left(\lambda^{2}-u^{2}\right)^{2} \\
& \Delta_{36}=\left(\frac{u-\lambda}{u+\lambda}\right)^{\frac{1}{2 \lambda}}\left(\lambda^{2}-u^{2}\right)\left(u^{2}-\lambda^{2}+x u_{x}\right) \\
& \Delta_{37}=-\left(\frac{u-\lambda}{u+\lambda}\right)^{\frac{1}{2 \lambda}}\left(\lambda^{2}-u^{2}\right) u_{x} \\
& \Delta_{45}=-\left(\frac{u-\lambda}{u+\lambda}\right)^{\frac{1}{2 \lambda}}\left(\left(\lambda^{2}-u^{2}\right) x u_{x}-\left(u^{2}-\lambda^{2}\right)^{2}+x^{2} u_{x}^{2} \frac{1-\lambda^{2}+2 u+2 u^{2}+2 u \lambda^{2}}{1+u^{2}}\right) \\
& \Delta_{46}=-\Delta_{37}
\end{aligned}
$$

${ }^{8}$ We use the symbolism $\Delta_{i j}$ to mean the determinant of the matrix which has $X_{i}$ and $X_{j}$ in the second and third rows respectively. 


$$
\begin{aligned}
\Delta_{47} & =\left(\frac{u-\lambda}{u+\lambda}\right)^{\frac{1}{2 \lambda}} u_{x}^{2} \frac{1-\lambda^{2}+2 u+2 u^{2}+2 u \lambda^{2}}{1+u^{2}} \\
\Delta_{48} & =\left(\frac{u-\lambda}{u+\lambda}\right)^{\frac{1}{2 \lambda}} u_{x}\left(\lambda^{2}-u^{2}+x u_{x} \frac{1-\lambda^{2}+2 u+2 u^{2}+2 u \lambda^{2}}{1+u^{2}}\right) \\
\Delta_{56} & =-\Delta_{13} \\
\Delta_{57} & =\Delta_{14} \\
\Delta_{67} & =-\Delta_{24} \\
\Delta_{68} & =-\Delta_{14} \\
\Delta_{78} & =\Delta_{24} .
\end{aligned}
$$

As a consequence the following are first integrals ${ }^{9}$ of system (2.17) and consequently of equation (2.11):

$$
\begin{aligned}
& I_{1}=\frac{\Delta_{13}}{\Delta_{14}}=\frac{u^{2}-\lambda^{2}+x u_{x}}{u_{x}} \\
& I_{2}=\frac{\Delta_{13}}{\Delta_{15}}=\left(\frac{u-\lambda}{u+\lambda}\right)^{\frac{1}{2 \lambda}} \frac{\lambda^{2}-u^{2}}{u^{2}-\lambda^{2}+x u_{x}} \\
& I_{3}=\frac{\Delta_{13}}{\Delta_{17}}=\left(\frac{u-\lambda}{u+\lambda}\right)^{\frac{1}{2 \lambda}} \frac{\left(\lambda^{2}-u^{2}\right)\left(u^{2}-\lambda^{2}+x u_{x}\right)}{u_{x}^{2}}=I_{2} I_{1}^{2} \\
& I_{4}=\frac{\Delta_{13}}{\Delta_{18}}=\left(\frac{u-\lambda}{u+\lambda}\right)^{\frac{1}{2 \lambda}} \frac{\lambda^{2}-u^{2}}{u_{x}}=I_{2} I_{1} \\
& I_{5}=\frac{\Delta_{14}}{\Delta_{15}}=-\frac{I_{2}}{I_{1}} \\
& I_{6}=\frac{\Delta_{14}}{\Delta_{17}}=I_{2} \\
& I_{7}=\frac{\Delta_{14}}{\Delta_{18}}=\frac{\Delta_{13}}{\Delta_{15}}=I_{2} \\
& I_{9}=\frac{\Delta_{15}}{\Delta_{17}}=-I_{1}^{2} .
\end{aligned}
$$

Naturally we have omitted all the other possible combinations (seventy-eight in total).

\section{A novel application}

If one looks for a suitable number ${ }^{10}$ of Lie symmetries admitted by a system of $n$th firstorder ordinary differential equations ${ }^{11}$ such as (2.8), then the following strategies may be used in the given order:

${ }^{9}$ We recall that $(2.11)$ possesses just two functionally independent first integrals and naturally the listed integrals are functions of these.

${ }^{10}$ It would be ideal to find $n$ Lie symmetries, although then we have to find an ad hoc method if the corresponding Lie algebra is not solvable [22], [7].

${ }^{11}$ Any ordinary differential equation of order $n$ can be trivially transformed into a system of $n$ firstorder ordinary differential equations. Therefore, if a single $n$ th-order equation does not possess Lie point symmetries, then we have to turn to any "equivalent" system of first-order equations, which possesses an infinite number of Lie point symmetries. 
1. Eliminate in turn each of the variables $w_{i}$ - if feasible - in order to obtain an equivalent $n$ th-order system which contains a single equation of second order and $n-2$ equations of first order. The admitted Lie symmetry algebra is no longer infinite-dimensional and the Lie group analysis can be deterministically applied ([43], [57], [49], [15], [53]). A secondary outcome of this strategy is that first integrals can also be determined ([39], [44], [35]).

2. Decrease the order of system (2.8) by one choosing in turn one of the variables $w_{i}$ as the new independent variable. Then apply either Strategy 1 or 3 in this list. For example, if $w_{1} \equiv y$ is the new independent variable, then system (2.8) becomes

$$
\frac{\mathrm{d} w_{k}}{\mathrm{~d} y}=\frac{W_{k}}{W_{1}} \equiv \Omega_{k}\left(y, w_{2}, \ldots, w_{n}\right) \quad(k=2, n) .
$$

This method has been illustrated in several papers (e.g. [47], [35], [45]) since its first instance, namely the Kepler problem [43].

3. Increase the order by using the transformation suggested by the Jacobi last multiplier, i.e. introduce a new dependent variable $R$ such that

$$
\dot{R}=\sum_{i=1}^{n} \frac{\partial W_{i}}{\partial w_{i}}
$$

and eliminate in turn - if feasible - each $w_{i}$ which appears in (3.2). Then system (2.8) transforms into a system of a single second-order and $n-1$ first-order equations. If a new independent variable was first chosen (Strategy 2), say $w_{1} \equiv y$, then $R$ is assumed to satisfy

$$
\frac{\mathrm{d} R}{\mathrm{~d} y}=\sum_{k=2}^{n} \frac{\partial \Omega_{k}}{\partial w_{k}}
$$

and then the strategy goes as above.

The reminder of this Section is dedicated to illustrate Strategy 3, although equation (3.42) is an example of Strategy 1.

\subsection{An equation from Kamke}

We consider the following equation [30]

$$
y^{\prime \prime}=\frac{y^{\prime 2}}{y}+f^{\prime}(x) y^{p+1}+p f(x) y^{\prime} y^{p},
$$

where $p \neq 0$ is a real constant and $f \neq 0$ is an arbitrary function of the independent variable $x$. This equation does not possess Lie point symmetries for general $f(x)$ and yet is trivially integrable [17]. We show that, if we increase the order of the corresponding system of first-order equations using the Jacobi last multiplier, then we find enough Lie point symmetries to enable us to integrate equation (3.4) à la Lie. Note that equation (3.4) has been cited as the prototype of a solvable equation with no Lie symmetries ([17], [35]). 
Equation (3.4) can be trivially transformed into a system of two first-order differential equations, i.e.,

$$
\begin{aligned}
& w_{1}^{\prime}=w_{2}, \\
& w_{2}^{\prime}=\frac{1}{w_{1}}\left(w_{1}^{p+2} f^{\prime}+w_{2}^{2}+w_{1}^{p+1} w_{2} f p\right),
\end{aligned}
$$

with $w_{1}=y$ and $w_{2}=y^{\prime}$ the two new unknowns in the independent variable $x$. The Jacobi last multiplier of system (3.5) has to satisfy equation (2.7), i.e.,

$$
\frac{\mathrm{d} \log (M)}{\mathrm{d} x}+p w_{1}^{p} f+2 \frac{w_{2}}{w_{1}}=0
$$

which suggests the transformation ${ }^{12} w_{2}=w_{1}\left(r_{2}^{\prime}-p w_{1}^{p} f\right) / 2$. Then system (3.5) transforms into a system of one second-order and one first-order differential equation in the two unknowns, $r_{2}$ and $w_{1}$, i.e.,

$$
\begin{aligned}
w_{1}^{\prime} & =\frac{w_{1}}{2}\left(r_{2}^{\prime}-w_{1}^{p} f p\right), \\
r_{2}^{\prime \prime} & =\frac{w_{1}^{p}}{2}\left(\left(r_{2}^{\prime}-w_{1}^{p} f p\right) f p+2 f^{\prime}\right)(p+2) .
\end{aligned}
$$

If we apply Lie group analysis to this system ${ }^{13}$, then we obtain a determining equation of parabolic type for $V\left(x, w_{1}, r_{2}\right)$ in two independent variables [39]. Its characteristic curve is $w_{1} e^{-r_{2} / 2}$ which yields the transformation, $w_{1}=r_{1} e^{r_{2} / 2}$, with $r_{1}$ a new unknown function of $x$. Then system (3.6) is transformed into:

$$
\begin{aligned}
r_{1}^{\prime} & =-\frac{1}{2}\left(e^{\frac{r_{2}}{2}} r_{1}\right)^{p} f p r_{1}, \\
r_{2}^{\prime \prime} & =\frac{\left(e^{\frac{r_{2}}{2}} r_{1}\right)^{p}}{2}\left(2 p f^{\prime}+4 f^{\prime}-\left(e^{\frac{r_{2}}{2}} r_{1}\right)^{p}(p+2) f^{2} p^{2}+p f(p+2) r_{2}^{\prime}\right),
\end{aligned}
$$

which admits a two-dimensional Lie symmetry algebra, $L_{2}$, generated by

$$
\Gamma_{1}=r_{1} \partial_{r_{1}}-2 \partial_{r_{2}}, \quad \Gamma_{2}=\frac{1}{r_{1}} \partial_{r_{1}}-\frac{2(p+2)}{p r_{1}^{2}} \partial_{r_{2}} .
$$

A basis of differential invariants of $L_{2}$ of order $\leq 1$ is given by:

$$
\tilde{x}=x, \quad \tilde{u}=r_{2}^{\prime}-(2+p) p f r_{1}^{p} e^{\frac{p r_{2}}{2}} .
$$

Then system (3.7) can be easily reduced to the following first-order differential equation:

$$
\frac{\mathrm{d} \tilde{u}}{\mathrm{~d} \tilde{x}}=0
$$

that yields the trivial general solution $\tilde{u}=a_{1}$. Then we use transformation (3.9) to obtain $r_{1}$ in terms of $r_{2}$ and $r_{2}^{\prime}$, i.e.,

$$
r_{1}=e^{-\frac{r_{2}}{2}}\left(\frac{r_{2}^{\prime}-a_{1}}{f(p+2)}\right)^{\frac{1}{p}} .
$$

\footnotetext{
${ }^{12}$ Of course $r_{2} \neq M$. It is not necessary for $r_{2}$ to be exactly equal to $M$ provided there is a point transformation between $r_{2}$ and $M$.

${ }^{13} \mathrm{We}$ look for Lie operators of the form $\Gamma=V\left(x, w_{1}, r_{2}\right) \partial_{x}+G_{1}\left(x, w_{1}, r_{2}\right) \partial_{w_{1}}+G_{2}\left(x, w_{1}, r_{2}\right) \partial_{r_{2}}$.
} 
Finally we have to solve the following second-order equation in the dependent variable $r_{2}$ :

$$
r_{2}^{\prime \prime}=-\left(a_{1}-r_{2}^{\prime}\right) \frac{\left(a_{1} p+2 r_{2}^{\prime}\right) p f+2(p+2) f^{\prime}}{2(p+2) f}
$$

which admits the two-dimensional nonabelian and intransitive Lie symmetry algebra generated by

$$
X_{1}=\partial_{r_{2}}, \quad X_{2}=e^{p \frac{r_{2}-a_{1} x}{p+2}} \partial_{r_{2}} .
$$

Following Lie's classification of two-dimensional algebras in the real plane [37] we have to transform the symmetries into the canonical forms

$$
\partial_{\tilde{r}_{2}}, \quad \tilde{r}_{2} \partial_{\tilde{r}_{2}}
$$

with $\bar{x}$ and $\tilde{r}_{2}$ the new independent and dependent variables, respectively. It is easy to derive that

$$
\bar{x}=x, \quad \tilde{r}_{2}=-\frac{p+2}{p} \exp \left[\frac{p}{p+2}\left(a_{1} x-r_{2}\right)\right]
$$

and equation (3.12) becomes

$$
\frac{\mathrm{d}^{2} \tilde{r}_{2}}{\mathrm{~d} \bar{x}^{2}}=\frac{\mathrm{d} \tilde{r}_{2}}{\mathrm{~d} \bar{x}} \frac{2 f^{\prime}+a_{1} p f}{2 f} .
$$

After two quadratures the general solution is obtained to be

$$
\tilde{r}_{2}=a_{2} \int f e^{\frac{a_{1} p x}{2}} d x+a_{3}
$$

and consequently the general solution of $(3.12)$ is

$$
r_{2}=-(p+2) \log \left(-\frac{p}{p+2}\left(a_{2} \int e^{\frac{a_{1} p x}{2}} f d x-a_{3}\right)\right)+a_{1} x .
$$

Finally the general solution of equation (3.4) is

$$
y=w_{1}=r_{1} e^{r_{2} / 2}=e^{\frac{x a_{1}}{2}}\left(-p \int e^{\frac{x a_{1} p}{2}} f d y+\tilde{a}_{2} p\right)^{-\frac{1}{p}}
$$

with $\tilde{a}_{2}=-a_{3} / a_{2}$.

\subsection{Darboux-Brioschi-Halphen system}

We now consider the Darboux-Brioschi-Halphen system which was first described in [12], then separately derived in [5] and solved in [19]:

$$
\begin{aligned}
& \dot{w}_{1}=w_{3} w_{2}-w_{1} w_{3}-w_{1} w_{2} \\
& \dot{w}_{2}=w_{1} w_{3}-w_{2} w_{1}-w_{2} w_{3} \\
& \dot{w}_{3}=w_{2} w_{1}-w_{3} w_{2}-w_{3} w_{1} .
\end{aligned}
$$


The Darboux-Brioschi-Halphen system appears in a variety of physical contexts, see [20] and [10] and the references therein.

The Jacobi last multiplier of system (3.20) is given by (2.9), i.e.,

$$
M=\exp \left(-2 \int\left(w_{1}+w_{2}+w_{3}\right) d t\right)
$$

which suggests the introduction of a new dependent variable, $R_{1}$, a function of $t$, such that

$$
\dot{R}_{1}=w_{1}+w_{2}+w_{3}
$$

and elimination of $w_{1}$, i.e. $w_{1}=\dot{R}_{1}-w_{2}-w_{3}$. Note that $W=2 \int R_{1} d t$ yields the Chazy equation [8], [10]:

$$
\dddot{W}=2 W \ddot{W}-3 \dot{W}^{2} .
$$

Then system (3.20) becomes a system of one second-order and two first-order differential equations in the three unknowns $R_{1}, w_{2}, w_{3}$. We apply Lie group analysis to this system $^{14}$ and obtain a determining equation of parabolic type for $V\left(t, R_{1}, w_{2}, w_{3}\right)$ in three independent variables [39]. Its characteristic curves suggest the following transformation

$$
w_{2}=r_{2}-w_{3}, \quad w_{3}=r_{3} e^{-2 R_{1}}+\frac{r_{2}}{2} .
$$

with $r_{2}$ and $r_{3}$ new unknown functions of $t$. Then system (3.20) is transformed into:

$$
\begin{aligned}
& \ddot{u}_{1}=\frac{3 e^{4 u_{1}} u_{2}^{2}-4 e^{4 u_{1}} u_{2} \dot{u}_{1}+4 u_{3}^{2}}{4 e^{4 u_{1}}} \\
& \dot{u}_{2}=\frac{-e^{4 u_{1}} u_{2}^{2}+4 u_{3}^{2}}{2 e^{4 u_{1}}} \\
& \dot{u}_{3}=2 u_{2} u_{3}
\end{aligned}
$$

with $u_{1} \equiv R_{1}, u_{2} \equiv r_{2}, u_{3} \equiv r_{3}$. This system admits a four-dimensional Lie symmetry algebra, $L_{4}$, which in the original unknowns $w_{1}, w_{2}, w_{3}$ becomes a three-dimensional Lie symmetry algebra $L_{3}$ isomorphic to $s l(2, \mathbb{R})$ generated by the following operators:

$$
\begin{aligned}
& X_{1}=\partial_{t}, \\
& X_{2}=t \partial_{t}-w_{1} \partial_{w_{1}}-w_{2} \partial_{w_{2}}-w_{3} \partial_{w_{3}}, \\
& X_{3}=-\frac{1}{2} t^{2} \partial_{t}+\left(w_{1} t-\frac{1}{2}\right) \partial_{w_{1}}+\left(w_{2} t-\frac{1}{2}\right) \partial_{w_{2}}+\left(w_{3} t-\frac{1}{2}\right) \partial_{w_{3}} .
\end{aligned}
$$

Note that, if we evaluate the determinant of matrix (2.6) corresponding to these three Lie symmetries, then we find that a Jacobi last multiplier of system (3.20) is given in closed form by:

$$
M_{1}=\frac{1}{\left(w_{1}-w_{2}\right)\left(w_{1}-w_{3}\right)\left(w_{2}-w_{3}\right)} .
$$

${ }^{14} \mathrm{We}$ look for Lie operators of the form $\Gamma=V\left(t, R_{1}, w_{2}, w_{3}\right) \partial_{t}+G_{1}\left(t, R_{1}, w_{2}, w_{3}\right) \partial_{R_{1}}+$ $G_{2}\left(t, R_{1}, w_{2}, w_{3}\right) \partial_{w_{2}}+G_{3}\left(t, R_{1}, w_{2}, w_{3}\right) \partial_{w_{3}}$. 


\subsection{A SIS system}

In [49] a simple epidemiological model was examined from the viewpoint of both Lie and Painlevé. The model is a particular case of the classical SIS model introduced by Kermack and McKendrick [31]. It was assumed that recovery from the nonfatal infective disease does not confer immunity. The two first-order ordinary differential equations are [4]

$$
\begin{aligned}
\dot{S} & =-\beta S I-\mu S+\gamma I+\mu K \\
\dot{I} & =\beta S I-(\mu+\gamma) I,
\end{aligned}
$$

in which the overdot denotes differentiation with respect to time, $S(t)$ is the susceptible component of the population, $I(t)$ is the infected component of the population, $\mu K$ represents a constant birth rate, $\mu$ is the proportionate death rate, $\beta$ is the infectivity coefficient of the typical Lotka-Volterra interaction term and $\gamma$ the recovery coefficient. The disease was assumed to be nonfatal so that the standard term removing deceased infectives ( $-\alpha I$ in Ref [4]) was omitted. In [49] a second-order ordinary differential equation was consequently obtained for $I$, viz.

$$
I \ddot{I}-\dot{I}^{2}+\beta I^{2} \dot{I}+\mu I \dot{I}+\beta \mu I^{3}+\mu(\mu+\gamma-\beta K) I^{2}=0,
$$

and, because this equation possesses only one trivial Lie point symmetry, hidden Lie symmetries were sought and successfully found by increasing the order of equation (3.27) by the transformation of Riccati type $I=\dot{w} /(\beta w)$. Here we show that the same result could have been achieved by using the Jacobi last multiplier.

The Jacobi last multiplier of system (3.26) suggests the following transformation

$$
S=\frac{\dot{r}_{1}+\beta I+\gamma+2 \mu}{\beta}
$$

which yields a system of one second-order and one first-order differential equations in the two unknowns $I$ and $r_{1}$. We apply Lie group analysis to this system ${ }^{15}$ and obtain a determining equation of parabolic type for $V\left(t, I, r_{1}\right)$ in two independent variables [39]. Its characteristic curve yields the transformation, $I=r_{2} e^{r_{1}}$, with $r_{2}$ a new unknown function. Then system (3.26) is transformed into:

$$
\begin{aligned}
& \dot{r}_{2}=r_{2}\left(e^{r_{1}} \beta r_{2}+\mu\right) \\
& \ddot{r}_{1}=-2 e^{2 r_{1}} \beta^{2} r_{2}^{2}-4 e^{r_{1}} \beta \mu r_{2}-2 e^{r_{1}} \beta r_{2} \dot{r}_{1}+\beta K \mu-\gamma \mu-2 \mu^{2}-\mu \dot{r}_{1}
\end{aligned}
$$

which admits a three-dimensional Lie symmetry algebra generated by ${ }^{16}$.

$$
\Gamma_{1}=\partial_{t}, \quad \Gamma_{2}=-\partial_{r_{1}}+r_{2} \partial_{r_{2}}, \quad \Gamma_{3}=e^{\mu t}\left(-\frac{2}{r_{2}} \partial_{r_{1}}+\partial_{r_{2}}\right) .
$$

\subsection{Initial value problems}

In [21] Hydon showed that initial-value problems may have more Lie symmetries than the underlying ordinary differential equations and presented an ad hoc method which uses

\footnotetext{
${ }^{15}$ We look for Lie operators of the form $\Gamma=V\left(t, I, r_{1}\right) \partial_{t}+G_{1}\left(t, I, r_{1}\right) \partial_{I}+G_{2}\left(t, I, r_{1}\right) \partial_{r_{1}}$.

${ }^{16}$ Of course $\Gamma_{1}$ is the trivial symmetry of (3.26) and $\Gamma_{2}$ is due to the transformation (3.28).
} 
Taylor series about $x=0$ in order to construct symmetries of a class of initial-value problems, namely third-order ordinary differential equations

$$
y^{\prime \prime \prime}=\omega\left(x, y, y^{\prime}, y^{\prime \prime}\right)
$$

subject to the initial condition

$$
y^{\prime \prime}(0)=0 .
$$

Here we consider the three examples given in [21] in order to show that Lie symmetries of the initial-value problem can be found by using the transformation suggested by the Jacobi last multiplier of the underlying equivalent system of first-order ordinary differential equations ${ }^{17}$. Furthermore we use the Jacobi last multiplier in order to find symmetries of another equation presented in [21] as open problems.

The first example in [21] is the Blasius equation, viz.

$$
y^{\prime \prime \prime}=y y^{\prime \prime},
$$

which can be trivially transformed into the following system of three first-order ordinary differential equations:

$$
\begin{aligned}
& w_{1}^{\prime}=w_{2}, \\
& w_{2}^{\prime}=w_{3}, \\
& w_{3}^{\prime}=w_{1} w_{3} .
\end{aligned}
$$

The Jacobi last multiplier suggests the transformation, $w_{1}=r_{1}^{\prime}$, with $r_{1}$ a new unknown function of $x$ which yields a system of one second-order and two first-order differential equation in the three unknowns $r_{1}, w_{2}$ and $w_{3}$, viz.

$$
\begin{aligned}
r_{1}^{\prime \prime} & =w_{2} \\
w_{2}^{\prime} & =w_{3} \\
w_{3}^{\prime} & =r_{1}^{\prime} w_{3} .
\end{aligned}
$$

We apply Lie group analysis to this system ${ }^{18}$ and obtain a determining equation of parabolic type for $V\left(x, r_{1}, w_{2}, w_{3}\right)$ in two independent variables [39]. Its characteristic curve yields the transformation, $w_{3}=r_{3} e^{r_{1}}$, with $r_{3}$ a new unknown function of $x$. Then system (3.35) is transformed into:

$$
\begin{aligned}
& r_{1}^{\prime \prime}=w_{2} \\
& w_{2}^{\prime}=e^{r_{1}} r_{3} \\
& r_{3}^{\prime}=0
\end{aligned}
$$

which means that $r_{3}$ is a constant, actually $r_{3}=y^{\prime \prime}(0)=0$ from condition (3.32). Then $w_{2}^{\prime}=0$, which implies $w_{2}=a_{1}$ a constant, and Lie group analysis applied to equation

\footnotetext{
${ }^{17}$ Actually in the third example one does not need to introduce the Jacobi last multiplier, the reduction method [43] being sufficient.

${ }^{18} \mathrm{We}$ look for Lie operators of the form $\Gamma=V\left(x, r_{1}, w_{2}, w_{3}\right) \partial_{x}+G_{1}\left(x, r_{1}, w_{2}, w_{3}\right) \partial_{r_{1}}+$ $G_{2}\left(x, r_{1}, w_{2}, w_{3}\right) \partial_{w_{2}}+G_{3}\left(x, r_{1}, w_{2}, w_{3}\right) \partial_{w_{3}}$.
} 
$r_{1}^{\prime \prime}=a_{1}$ yields an eight-dimensional Lie symmetry algebra. Note that, if $r_{3} \neq 0$, then the system of two equations (3.36a)-(3.36b) would have admitted just a two-dimensional trivial Lie point symmetry algebra.

A second example in [21] is:

$$
y^{\prime \prime \prime}=\frac{2 x}{y^{5}}-\frac{5 y^{\prime} y^{\prime \prime}}{y}
$$

which can be trivially transformed into the following system of three first-order ordinary differential equations:

$$
\begin{aligned}
& w_{1}^{\prime}=w_{2}, \\
& w_{2}^{\prime}=w_{3}, \\
& w_{3}^{\prime}=\frac{2 x}{w_{1}^{5}}-\frac{5 w_{2} w_{3}}{w_{1}} .
\end{aligned}
$$

The Jacobi last multiplier suggests the transformation, $w_{2}=-r_{2}^{\prime} w_{1} / 5$, with $r_{2}$ a new unknown function of $x$ which yields a system of one second-order and two first-order differential equations in the three unknowns $r_{2}, w_{1}$ and $w_{3}$. We apply Lie group analysis to this system and obtain a determining equation of parabolic type for $V\left(x, w_{1}, w_{3}, r_{2}\right)$ in three independent variables [39]. Its characteristic curves yield the transformations, $w_{1}=r_{1} e^{-r_{2} / 5}$ and $w_{3}=r_{3} e^{r_{2}}$, with $r_{1}$ and $r_{3}$ new unknown functions of $x$. Then system (3.38) is transformed into three separated equations, two of them being easy to solve:

$$
\begin{aligned}
& r_{1}^{\prime}=0 \quad \Rightarrow r_{1}=a_{1} \\
& r_{3}^{\prime}=\frac{2 x}{a_{1}^{5}} \quad \Rightarrow r_{3}=\frac{a_{1}^{5} a_{2}+x^{2}}{a_{1}^{5}}
\end{aligned}
$$

and a third equation

$$
r_{2}^{\prime \prime}=\frac{-25 \exp \left[\frac{6 r_{2}}{5}\right]\left(a_{1}^{5} a_{2}+x^{2}\right)+a_{1}^{6} r_{2}^{\prime 2}}{5 a_{1}^{6}} .
$$

Note that condition (3.32) implies $a_{2}=0$. In this case equation (3.40) admits a twodimensional Lie point symmetry algebra generated by:

$$
X_{1}=\frac{1}{5} x\left(-x \partial_{x}+5 \partial_{r_{2}}\right), \quad X_{2}=-\frac{3}{10} x \partial_{x}+\partial_{r_{2}}
$$

and thus can be solved à la Lie.

A third example in [21] is:

$$
y^{\prime \prime \prime}=y^{\prime \prime 2}+\left(\frac{y^{\prime}}{y}-x y\right) y^{\prime \prime}+y
$$

which can be trivially transformed into the following system of three first-order ordinary differential equations:

$$
\begin{aligned}
& w_{1}^{\prime}=w_{2}, \\
& w_{2}^{\prime}=w_{3},
\end{aligned}
$$




$$
w_{3}^{\prime}=w_{3}^{2}+\left(\frac{w_{2}}{w_{1}}-x w_{1}\right) w_{3}+w_{1} .
$$

Here we show how to obtain Lie symmetries by using just the reduction method [43]. If we derive $w_{2}$ from equation $(3.43 c)$, i.e.,

$$
w_{2}=\frac{w_{1}}{w_{3}}\left(w_{3}^{\prime}+x w_{1} w_{3}-w_{1}-w_{3}^{2}\right)
$$

then (3.43) transforms into a system of one second-order and one first-order equations in the unknowns $w_{1}, w_{3}$. We apply Lie group analysis to this system and obtain a determining equation of parabolic type for $V\left(x, w_{1}, w_{3}\right)$ in two independent variables [39]. Its characteristic curve yields the transformation, $w_{1}=r_{1} w_{3}$, with $r_{1}$ a new unknown function of $x$. Then equation (3.43a) is transformed into a Riccati equation, viz.

$$
r_{1}^{\prime}=r_{1}^{2}\left(x w_{3}-1\right)-r_{1} w_{3},
$$

which suggests the use of the transformation

$$
r_{1}=-\frac{R_{1}^{\prime}}{\left(x w_{3}-1\right) R_{1}}
$$

with $R_{1}$ a new unknown function of $x$, in order to obtain

$$
R_{1}^{\prime \prime}=R_{1}^{\prime} \frac{x w_{3}^{\prime}-w_{3}\left(x w_{3}-2\right)}{x w_{3}-1} .
$$

This can be easily integrated once to obtain

$$
R_{1}^{\prime}=a_{1}\left(x w_{3}-1\right) e^{-\int w_{3} d x} .
$$

This suggests the transformation $w_{3}=-r_{3}^{\prime} / r_{3}$ in order to remove the exponential of an integral. Then equation (3.47) is transformed into

$$
R_{1}^{\prime \prime}=R_{1}^{\prime} \frac{x r_{3}^{\prime \prime}+2 r_{3}^{\prime}}{x r_{3}^{\prime}+r_{3}}
$$

which is easy to integrate with two quadratures. Its general solution is:

$$
R_{1}=a_{1} x r_{3}+a_{2}
$$

Now we apply Lie group analysis to the third-order equation in $r_{3} \equiv u$, viz.

$$
\begin{aligned}
u^{\prime \prime \prime}= & \frac{1}{\left(a_{1} u x+a_{2}\right)^{2} a_{1} u}\left[\left(\left(3 u^{\prime} x^{2}+2 u^{\prime \prime}\right) u+\left(2 u^{\prime}+3 u^{\prime \prime} x\right) u^{\prime}\right) a_{1}^{2} a_{2} u+\left(3 a_{1} u^{\prime}+a_{2}\right) a_{2}^{2} u^{\prime}\right. \\
& \left.-\left(\left(\left(2 u^{\prime}-3 u^{\prime \prime} x\right) u+2 u^{\prime 2} x\right) u^{\prime} x-\left(\left(x^{3}-2\right) u^{\prime}+2 u^{\prime \prime} x\right) u^{2}\right) a_{1}^{3} u\right] .
\end{aligned}
$$

If $a_{2}=0$, then we obtain a four-dimensional Lie point symmetry algebra generated by

$$
\begin{aligned}
& X_{1}=u \log (u) \partial_{u}, \\
& X_{2}=u \partial_{u},
\end{aligned}
$$




$$
\begin{aligned}
& X_{3}=x u K_{\frac{2}{3}}\left(\frac{2}{3} x^{\frac{3}{2}}\right) \partial_{u}, \\
& X_{4}=x u I_{\frac{2}{3}}\left(\frac{2}{3} x^{\frac{3}{2}}\right) \partial_{u},
\end{aligned}
$$

with $K$ being the modified Bessel function of the second kind and $I$ the modified Bessel function of the first kind ${ }^{19}$. As far as we know, this is only the second appearance of special functions in the generators of Lie symmetries admitted by a nonlinear differential equation. The preceding example, which involves elliptic integrals, can be found in [55].

Another equation given in [21] is the following ${ }^{20}$ :

$$
y^{\prime \prime \prime}=\left(y-\frac{x^{2}}{2}\right)\left(y^{\prime \prime}-1\right)
$$

which can be trivially transformed into the following system of three first-order ordinary differential equations:

$$
\begin{aligned}
& w_{1}^{\prime}=w_{2}, \\
& w_{2}^{\prime}=w_{3}, \\
& w_{3}^{\prime}=\left(w_{1}-\frac{x^{2}}{2}\right)\left(w_{3}-1\right) .
\end{aligned}
$$

The Jacobi last multiplier suggests the transformation, $w_{1}=r_{1}^{\prime} / r_{1}+x^{2} / 2$, with $r_{1}$ a new unknown function of $x$ which yields a system of one second-order and two first-order differential equations in the three unknowns, $r_{1}, w_{2}$ and $w_{3}$. We apply Lie group analysis to this system and obtain a determining equation of parabolic type for $V\left(x, r_{1}, w_{2}, w_{3}\right)$ in two independent variables [39]. Its characteristic curve yields the transformation, $w_{3}=r_{1} r_{3}+1$, with $r_{3}$ a new unknown function of $x$. Then system (3.54) is transformed into the following system:

$$
\begin{aligned}
r_{1}^{\prime \prime} & =\frac{r_{1}^{\prime 2}}{r_{1}}+r_{1} w_{2}-x r_{1} \\
w_{2}^{\prime} & =r_{1} r_{3}+1 \\
r_{3}^{\prime} & =0 \quad \Rightarrow r_{3}=a_{0} .
\end{aligned}
$$

If $a_{0}=0$, then $w_{2}=x+a_{1}$ and $r_{1} \equiv u$ has to satisfy the following second-order ordinary differential equation:

$$
u^{\prime \prime}=\frac{u^{\prime 2}}{u}+a_{1} u
$$

which admits an eight-dimensional Lie symmetry algebra generated by the following operators:

$$
\begin{aligned}
& \Gamma_{1}=-\frac{1}{4}\left(-2 \log (u)+a_{1} x^{2}\right)\left(2 x \partial_{x}+\left(2 \log (u)+a_{1} x^{2}\right) u \partial_{u}\right) \\
& \Gamma_{2}=\left(-\frac{3}{2} a_{1} x^{2}+\log (u)\right) \partial_{x}-a_{1}^{2} x^{3} \partial_{u}
\end{aligned}
$$

${ }^{19}$ We used Maple 9 to find the general solution of the determining equation $-x^{2} s^{\prime \prime}(x)+2 x s^{\prime}(x)+\left(x^{3}-\right.$ 2) $s(x)=0$.

${ }^{20}$ It comes from Blasius boundary layer theory. 


$$
\begin{aligned}
& \Gamma_{3}=\frac{1}{2} x\left(2 x \partial_{x}+\left(2 \log (u)+a_{1} x^{2}\right) u \partial_{u}\right) \\
& \Gamma_{4}=-\frac{1}{2} u\left(-2 \log (u)+a_{1} x^{2}\right) \partial_{u} \\
& \Gamma_{5}=\partial_{x} \\
& \Gamma_{6}=x\left(\partial_{x}+a_{1} x u \partial_{u}\right) \\
& \Gamma_{7}=u \partial_{u} \\
& \Gamma_{8}=x u \partial_{u} .
\end{aligned}
$$

This means that equation (3.56) is linearizable [37] by means of a point transformation. In order to find the linearizing transformation we have to look for a two-dimensional abelian intransitive subalgebra and, following Lie's classification of two-dimensional algebras in the real plane [37], we have to transform it into the canonical form (2.13). We find that one such subalgebra is that generated by $\Gamma_{1}$ and $\Gamma_{3}$. Then it is easy to derive that

$$
\tilde{x}=-\frac{2 x}{-2 \log (u)+a_{1} x^{2}}, \quad \tilde{u}=\frac{2}{-2 \log (u)+a_{1} x^{2}}
$$

and equation (3.56) becomes

$$
\frac{\mathrm{d}^{2} \tilde{u}}{\mathrm{~d} \tilde{x}^{2}}=0
$$

which trivially yields $\tilde{u}=b_{1} \tilde{x}+b_{2}$ with $b_{1}$ and $b_{2}$ arbitrary constants. Consequently the general solution of (3.56) is

$$
u=\exp \left(\frac{b_{2} a_{1} x^{2}-2 b_{1} x-2}{2 b_{2}}\right)
$$

and finally

$$
y=w_{1}=\frac{r_{1}^{\prime}}{r_{1}}+\frac{x^{2}}{2}=a_{1} x-\frac{b_{1}}{b_{2}}+\frac{x^{2}}{2} .
$$

\section{References}

[1] Bartoccini F and Verdini S, Sui Congressi degli Scienziati. Edizioni dell'Ateneo, Roma, 1952.

[2] Berrone L R and Giacomini H, Inverse Jacobi multipliers, Rend. Circ. Mat. Palermo Serie II $\mathbf{5 2}$ (2003), 77-130.

[3] Bianchi L, Lezioni sulla teoria dei gruppi continui finiti di trasformazioni, Enrico Spoerri, Pisa, 1918.

[4] Brauer F, Basic ideas of mathematical epidemiology, in Mathematical Approaches for Emerging and Reemerging Infectious Diseases, (Castillo-Chavez C, Blower Sally, van den Driessche P, Kirschner D and Yakubu A A, Eds.), Springer-Verlag, New York, 2002, 31-65.

[5] Brioschi F, Sur un systéme d'équations différentielles, C. R. Acad. Sci. Paris 92 (1881), 1389-1393.

[6] Calogero F, On a modified version of a solvable ODE due to Painlevé, J. Phys. A: Math. Gen. 35 (2002), 985-992. 
[7] Cerquetelli T, Ciccoli N and Nucci M C, Four-dimensional Lie symmetry algebras and fourthorder ordinary differential equations, J. Nonlin. Math. Phys. 9-s2 (2002), 24-35.

[8] Chazy J, Sur les équations différentielles du troisième ordre et d'ordre supérieur dont l’intégrale générale a ses points critiques fixes, Acta Math. 34 (1910), 317-385.

[9] Chella T, Vantaggi che si possono trarre da noti invarianti integrali e differenziali in alcuni problemi di integrazione, Ann. R. Scuola Normale Sup. Pisa Sc. Fis. Mat. Nat. 11 (1910), 9-137.

[10] Clarkson P A and Olver P J, Symmetry and the Chazy equation, J. Diff. Eqn. 124 (1996), 225-246.

[11] Clarkson P A and Cosgrove C M, Symmetry, the Chazy equation and Chazy hierarchies, in Isomonodromic Deformations and Applications in Physics, Editors: Harnad J and Its A R, CMR Proceedings and Lecture Notes, Montréal, 2002, 113-129.

[12] Darboux G, Sur la théorie des coordinneés curvilignes et les systémes orthogonaux, Ann. Ec. Norm. Supér. 7 (1878), 101-150.

[13] De Donder T, Sur le multiplicateur de Jacobi généralisé, Bull. de l'Acad. Roy. de Belgique, Cl. de Sciences (1908), 795-811.

[14] Deshpande S M, The averaging principle and the diagram technique, Physica A 80 (1975), 287-299.

[15] Edwards M and Nucci M C, Application of Lie group analysis to a core group model for sexually transmitted diseases, (2005) (submitted).

[16] González-Gascón F and González-López A, Symmetries of differential equations. IV. J. Math. Phys. 24 (1983), 2006-2021.

[17] González-López A, Symmetry and integrability by quadratures of ordinary differential equations, Phys. Lett. A 133 (1988), 190-194.

[18] Grammaticos B, Moulin Ollagnier J, Ramani A, Strelcyn J M and Wojciechowski S, Integrals of quadratic ordinary differential equations in $R^{3}$ : the Lotka-Volterra system, Physica $A \mathbf{1 6 3}$ (1990), 683-722.

[19] Halphen G H, Sur un systéme d'équations différentielles orthogonaux, C. R. Acad. Sci. Paris 92 (1881), 1101-1103.

[20] Harnad J and McKay J, Modular solutions to equations of generalized Halphen type, Proc. R. Soc. Lond. A 456 (2000), 261-294.

[21] Hydon P E, Symmetry analysis of initial-value problems, J. Math. Anal. Appl. (2004), submitted.

[22] Ibragimov N H and Nucci M C, Integration of third-order ordinary differential equations by Lie's method: equations admitting three-dimensional Lie algebras, Lie Groups and Their Applications 1 (1994), 49-64.

[23] Ince E L, Ordinary Differential Equations. Dover, New York, 1956.

[24] Jacobi C G J, Sur un noveau principe de la mécanique analytique, C. R. Acad. Sci. Paris 15 (1842), 202-205. 
[25] Jacobi C G J, Sul principio dell'ultimo moltiplicatore, e suo uso come nuovo principio generale di meccanica, Giornale Arcadico di Scienze, Lettere ed Arti 99 (1844), 129-146.

[26] Jacobi C G J, Theoria novi multiplicatoris systemati æquationum differentialium vulgarium applicandi, J. Reine Angew. Math. 27 (1844), 199-268.

[27] Jacobi C G J, Theoria novi multiplicatoris systemati æquationum differentialium vulgarium applicandi, J. Reine Angew. Math. 29 (1845), 213-279 and 333-376.

[28] Jacobi C G J, Vorlesungen über Dynamik. Nebst fünf hinterlassenen Abhandlungen desselben herausgegeben von A. Clebsch, Druck und Verlag von Georg Reimer, Berlin, 1886.

[29] Jones S E and Vujanović B, On the inverse Lagrangian problem, Acta Mech. 73 (1988), $245-251$.

[30] Kamke E, Differentialgleichungen Lösungsmethoden und Lösungen, Chelsea, New York, 1974.

[31] Kermack W O and McKendrick A G, A contribution to the mathematical theory of epidemics, Proc. Roy. Soc. London 115 (1927), 700-721.

[32] Koenigsberger L, Carl Gustav Jacob Jacobi, Teubner, Leipzig, 1904.

[33] Krishna Rao D, The last multiplier in quantum mechanics, J. Mysore Univ. Sect. B. 2 (1941), $1-4$.

[34] Laguerre E N, Application du principe du dernier multiplicateur à l'intégration d'une équation différentielle du second ordre, Bull. Sci. Math. Astron. 2 (1871), 246-250.

[35] Leach P G L and Nucci M C, Reduction of the classical MICZ-Kepler problem to a twodimensional linear isotropic harmonic oscillator, J. Math. Phys. 45 (2004), 3590-3604.

[36] Lie S, Veralgemeinerung und neue Verwerthung der Jacobischen Multiplicator-Theorie, Christ. Forh. (1874), 198-226.

[37] Lie S, Vorlesungen über Differentialgleichungen mit bekannten infinitesimalen Transformationen, Teubner, Leipzig, 1912.

[38] Madhava Rao B S, On the reduction of dynamical equations to the Lagrangian form, Proc. Benares Math. Soc. 2 (1940), 53-59.

[39] Marcelli M and Nucci M C, Lie point symmetries and first integrals: the Kowalevsky top, $J$. Math. Phys. 44 (2003), 2111-2132.

[40] Noether E, Invariante Variationsprobleme, Nachr d König Gesellsch d Wiss zu Göttingen, Math-phys Klasse (1918), 235-257 (English translation: Noether E and Tavel M A, Invariant variation problems, Transport Theory and Statistical Mechanics 1 (1971), 183-207).

[41] Nucci M C, Interactive REDUCE programs for calculating classical, non-classical and LieBäcklund symmetries of differential equations, Preprint Georgia Tech Math 062090-051, 1990.

[42] Nucci M C, Interactive REDUCE programs for calculating Lie point, non-classical, LieBäcklund, and approximate symmetries of differential equations: manual and floppy disk, in CRC Handbook of Lie Group Analysis of Differential Equations. Vol. 3: New Trends in Theoretical Developments and Computational Methods, Editor: Ibragimov N H, CRC Press, Boca Raton, 1996, 415-481. 
[43] Nucci M C, The complete Kepler group can be derived by Lie group analysis, J. Math. Phys. 37 (1996), 1772-1775.

[44] Nucci M C, Lorenz integrable system moves à la Poinsot, J. Math. Phys. 44 (2003), 41074118.

[45] Nucci M C, Calogero's "goldfish" is indeed a school of free particles, J. Phys. A: Math. Gen. 37 (2004), 11391-11400.

[46] Nucci M C, Jacobi's three-body system moves like a free particle, J. Nonlin. Math. Phys. 12-s1 (2005), 499-506.

[47] Nucci M C and Leach P G L, The harmony in the Kepler and related problems, J. Math. Phys. 42 (2001), 746-764.

[48] Nucci M C and Leach P G L, Jacobi's last multiplier and the complete symmetry group of the Euler-Poinsot system, J. Nonlin. Math. Phys. 9-s2 (2002), 110-121.

[49] Nucci M C and Leach P G L, An integrable S-I-S model, J. Math. Anal. Appl. 290 (2004), 506-518.

[50] Nucci M C and Leach P G L, Jacobi's last multiplier and symmetries for the Kepler problem plus a lineal story, J. Phys. A: Math. Gen. 37 (2004), 7743-7753.

[51] Opatowski I, Two-dimensional compressible flows, Proc. Symposia Appl. Math. (A.M.S.) 1 (1949), 87-93.

[52] Painlevé P, Mémoire sur les équations différentielles dont l'intégral est uniforme, Bull. Soc. Mat. France 28 (1900), 201-261.

[53] Rellini M and Nucci M C, Application of Lie group analysis to a SIRI disease transmission model (2005) (in preparation).

[54] Roman-Miller L and Broadbridge P, Exact integration of reduced Fisher's equation, reduced Blasius equation, and the Lorenz model, J. Math. Anal. Appl. 251 (2000), 65-83.

[55] Rosati L and Nucci M C, A Lie symmetry connection between Jacobi modular equation and Schwarzian equation, J. Nonlin. Math. Phys. 12 (2005) (this issue).

[56] Saltykow N, Lie-ovo generalizanje teorije poslednjeg množitelja, Glas. Srpske Akad. Nauka. Od. Prirod.-Mat. Nauka 198 (1950), 1-16.

[57] Torrisi V and Nucci M C, Application of Lie group analysis to a mathematical model which describes HIV transmission, in The Geometrical Study of Differential Equations, Editors: Leslie J A and Hobart T P, American Mathematical Society, Providence, 2001, 31-40.

[58] Whittaker E T, A Treatise on the Analytical Dynamics of Particles and Rigid Bodies, Dover, New York, 1944. 